Acta Crystallographica Section A

Foundations of Crystallography

ISSN 0108-7673

Received 17 October 2009

Accepted 11 November 2009

(C) 2010 International Union of Crystallography

Printed in Singapore - all rights reserved

\section{The new age of structural dynamics}

\author{
Ahmed H. Zewail \\ Physical Biology Center for Ultrafast Science and Technology, Arthur Amos Noyes Laboratory of \\ Chemical Physics, California Institute of Technology, Pasadena, CA 91125, USA. Correspondence \\ e-mail:zewail@caltech.edu
}

A perspective on the new developments of structural dynamics is presented. The scope of applications and advances, from microscopy to diffraction and spectroscopy, defines the new age of endeavour.
For decades, the advent of tools such as electron and neutron diffraction, X-ray absorption and diffraction, NMR spectroscopy and electron microscopy have enabled the direct probing of the time-averaged, static structure of matter. The impact has been wide ranging in physics, chemistry and biology. It is now possible to determine three-dimensional structures, with atomic scale resolution, in systems ranging from small molecules to crystals, and from DNA and proteins to viruses and particles. The latest is the work on the structure of the ribosome protein-making machine which was awarded the 2009 Nobel Prize in Chemistry (see Fig. 1). However, a full description of biological functions, chemical reactions or phase transitions requires real-time visualization of the actual events, i.e. the ability to follow a sequence of steps characterizing a given process. Such changes occur on different timescales through transition states and intermediates on a complex energy landscape.

The elementary steps for molecular transformations involve motion of atoms on a timescale of femtoseconds and a lengthscale of ångströms. Molecular-scale motions are slower, typi-

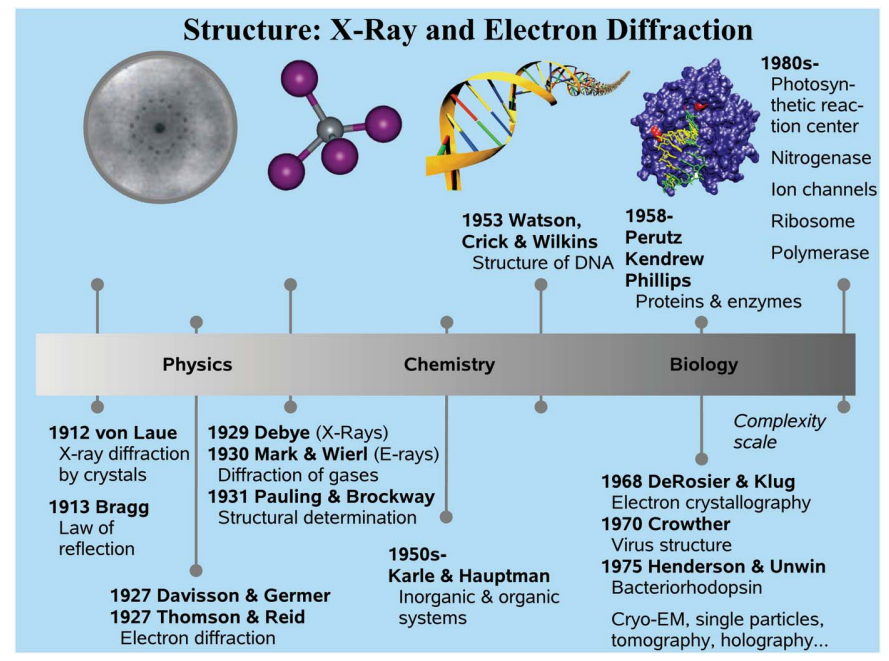

Figure 1

Developments in structural determination, from sodium chloride crystals to DNA and proteins, including the Nobel Prizes awarded (date given is that of development). Adapted from Zewail (2006). cally on the picosecond to microsecond scale. In solids, the velocity of sound is $300-1000 \mathrm{~m} \mathrm{~s}^{-1}$ and the ranges of timeand length-scales vary from femtoseconds to nanoseconds, and from ångströms to microns. Such scales are determined by the nature of the processes involved, and capturing the events as they unfold provides knowledge of the mechanism hidden in time-averaged measurements. Moreover, the timescale is directly telling of dynamics of the atomic motions which may involve the crossing of an energy barrier, bond making and breaking or coherent collective phenomena.

With the advent of femtosecond lasers, it has become possible to observe atomic motions during the change on a timescale that is shorter than a single vibrational period. The key to this atomic scale resolution lies in the ability to prepare a coherent state and in the (controlled) probing of motion in real time. Over the years, the field of femtochemistry [for a review see Zewail (2000), originally published in Frängsmyr (2000)] has encompassed studies in different phases (gas phase, crystalline and amorphous structures, and solutions) with extensions to adjacent areas in femtobiology (see Fig. 2).

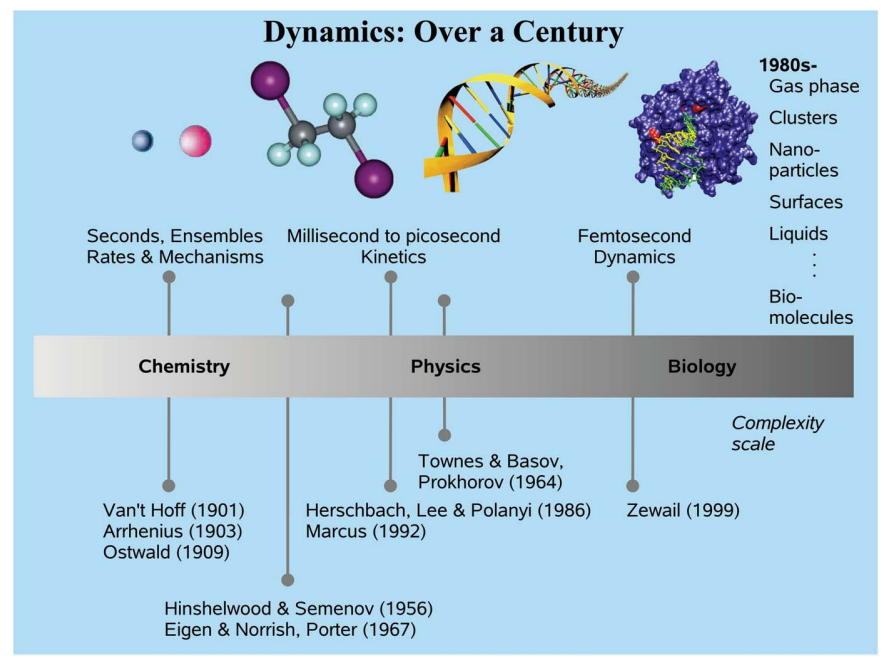

Figure 2

Developments in studies of kinetics and dynamics, from seconds to the femtosecond atomic scale as cited (date indicated) by Nobel Prizes awarded over the past century. Adapted from Zewail (2006). 
While the relationship between the spectroscopic observables (intensity and energy of transitions) and structure is rather clear in the case of small molecular systems, it becomes more ambiguous when the systems grow in complexity and size, as we must consider all atomic movements at once. A further degree of complexity is introduced when dealing with condensed phases or biological structures as, additionally, intermolecular coordinates with the solvent matrix come into play.

During the past decade, developments have focused on the possibility of mapping out three-dimensional structures in the act of change. Initially, with large-scale installations (synchrotrons) the collimated X-ray beams of high photon fluxes $\left(10^{3}\right.$ to $\left.10^{6}\right)$ which were used for the static structural determination of proteins were utilized with a time resolution of $\sim 100$ ps. This timescale is not sufficient to observe atomic motions. More recently, femtosecond sources became available, although with significantly lower fluxes (10-100 photons per pulse). To date, most of the studies, mainly by X-ray diffraction, have been on solid materials with the focus on lattice dynamics, melting and phase transitions. Progress has also been made in the use of coherent beams and inversion algorithms to obtain materials and biological images with typical resolutions of $\sim 100 \mathrm{~nm}$ and with resolutions approaching tens of $\mathrm{nm}$ for beams with high coherence properties (Zewail \& Thomas, 2009).

Electron microscopy, diffraction and spectroscopy have had a long history as powerful techniques for (static) structural determination and elemental analysis. When it comes to isolated molecular systems, nanometre-scale interfaces, and membrane proteins and single bioparticles, the strong electron-matter interaction is critical for providing the required sensitivity. Moreover, because the de Broglie wavelength reaches a picometre scale, and the beam can be focused with high precision, a resolution of less than an ångström can be achieved in real-space imaging. Four-dimensional ultrafast electron microscopy (4D UEM) with convergent-beam, nearfield and tomographic variants (Fig. 3) has recently been developed as a table-top methodology for real-space imaging, diffraction and electron-energy-loss spectroscopy with the time resolution being ten orders of magnitude better than hitherto. This development based on single-electron imaging now provides the means for atomic scale spatiotemporal resolutions, and applications currently span complex structures in chemical reactions, condensed media and biological assemblies.

This special issue provides selective studies that span different timescales, systems and phases. The compendium illustrates the scope of applications using X-ray absorption and diffraction, and electron diffraction. Electron crystallography and microscopy are not as well covered, but the

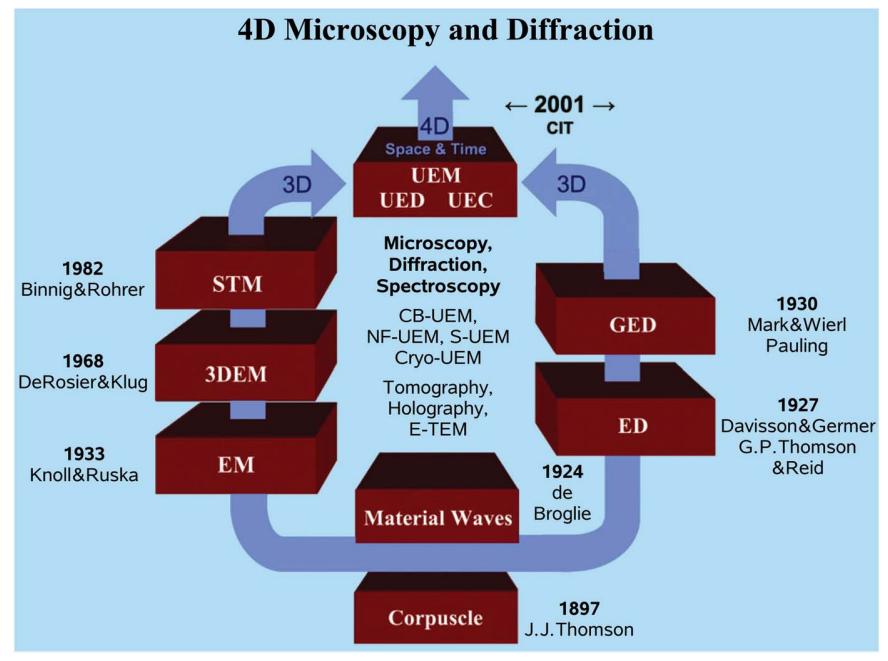

Figure 3

A brief history of developments in three- and four-dimensional imaging, microscopy and diffraction, displaying milestones, since the discovery of the electron (corpuscle) in 1897, to reach the atomic scale spatial (threedimensional) and spatiotemporal (four-dimensional) resolutions. Adapted from Zewail (2008).

reader is referred to recent publications [Zewail (2006) and references therein; Barwick et al. (2008); Zewail (2008); Barwick et al. (2009); Carbone et al. (2009); Yurtsever \& Zewail (2009); Shorokhov \& Zewail (2009)] and a comprehensive monograph (Zewail \& Thomas, 2009) to view the state of the art in the field. It is worth recalling that these powerful electron- and X-ray-based techniques were the force behind many achievements in static structural determination, and now, with atomic scale spatiotemporal resolution, the door is open for a new age in structural dynamics.

\section{References}

Barwick, B., Flannigan, D. J. \& Zewail, A. H. (2009). Nature (London), 462, 902-906.

Barwick, B., Park, H. S., Kwon, O.-H., Baskin, J. S. \& Zewail, A. H. (2008). Science, 322, 1227-1231.

Carbone, F., Kwon, O.-H. \& Zewail, A. H. (2009). Science, 325, 181184.

Frängsmyr, T. (2000). Editor. Les Prix Nobel: The Nobel Prizes 1999, pp. 103-203. Stockholm: Almqvist and Wiksell.

Shorokhov, D. \& Zewail, A. H. (2009). J. Am. Chem. Soc. 131, 1799818015.

Yurtsever, A. \& Zewail, A. H. (2009). Science, 326, 708-712.

Zewail, A. H. (2000). Angew. Chem. Int. Ed. 39, 2586-2631.

Zewail, A. H. (2006). Annu. Rev. Phys. Chem. 57, 65-103.

Zewail, A. H. (2008). Editor. Physical Biology: From Atoms to Medicine. London: Imperial College Press.

Zewail, A. H. \& Thomas, J. M. (2009). Four-Dimensional Electron Microscopy: Imaging in Space and Time. London: Imperial College Press. 